\title{
A experiência da liberdade como experiência da graça
}

\section{L'expérience de la liberté comme expérience de la grâce}

\author{
MARIE BAHUREL
}

\begin{abstract}
Résumé: L'articulation de la liberté et de la grâce est à la fois un problème classique de la philosophie morale et un enjeu central de la pensée de Gabriel Marcel. Le but de notre article est d'éclairer cette question à l'aide de la notion marcellienne d'attestation créatrice, qui est pour Gabriel Marcel le paradigme de tout acte libre. Dans l'acte libre s'accomplissent la reconnaissance d'un donné préalable à ma liberté qui fonctionne comme un appel, et une création par la réponse que j'apporte à cet appel. Que s'agit-il de reconnaître? Gabriel Marcel part de l'expérience la plus commune de l'acte libre, sans faire appel à une théologie de la grâce: je suis appelé à agir de telle ou telle façon selon la situation dans laquelle je suis placé, quelque chose me précède donc. Quant à la création, il s'agit d'abord d'une création de moi par moi: la liberté s'articule elle-même au mystère de l'être, par elle je suis de plus en plus. En cela, la liberté est pour Marcel "le mystère central de mon être", et explorer l'expérience de la liberté permet d'approcher le mystère ontologique. Or l'expérience que je fais de ma liberté dans des actes libres est une expérience de mon insertion dans des situations particulières: l'expérience de la liberté est indissociable de celle de ma propre incarnation. La pensée de Gabriel Marcel permet donc de penser notre liberté selon une voie qui échappe aux pièges du nihilisme (le tout-contingent), à l'illusion de la toutepuissance (le refus des limites liées à l'incarnation), et au dualisme qui aspire à la mort du corps. A partir de là, nous pouvons voir comment Gabriel Marcel articule expérience d'incarnation et reconnaissance d'une transcendance, à l'exact inverse d'un matérialisme réducteur.
\end{abstract}

Mots-clés: Liberté. Grâce. Témoignage créateur. Personne. Être.

Abstract: The articulation of freedom with grace is both a classic subject-matter of moral philosophy and a central concern of Gabriel Marcel's thought. This article aims at shedding some light on the question, using the Marcelian notion of a creative testimony which is, for Gabriel Marcel, the paradigm of any free act. In any free act occur the recognition of a prerequisite to our freedom which, in turn, acts the part of an appeal. Then comes a creation through the response we bring to this appeal. What do we have to reckon with here? Gabriel Marcel starts with the commonest experience of a free act, without using a theology of grace. We are meant to act in such way or another according to the situations we deal with, we are therefore always preceded by something. As for creation, it is first and foremost a creation of the self by the self. Indeed, freedom hinges on the mystery of being, I am more and more because of it. Henceforth, Marcel considers freedom as "the central mystery of our being", and experiencing freedom provides us with the opportunity to approach the ontological mystery. However, experiencing our freedom through our free acts comes down to experiencing our insertion in specific situations. Our experience of freedom is therefore inseparable from that of our own incarnation. Gabriel Marcel's thought leads us to approach our freedom in ways that avert the pitfalls of nihilism (and its endless contingency), the illusion of all-powerfulness (the denial of the limits pertaining to incarnation), and the dualism that aspires to the death of the body. Henceforth, we can see

\footnotetext{
${ }^{1}$ Professeur agrégée de philosophie, ancienne élève de l'Ecole Normale Supérieure (ENS Ulm). Enseigne dans les classes secondaires. Membre du conseil d'administration de l'Association Présence de Gabriel Marcel. A mené des travaux sur Simone Weil et Martin Buber. Poursuit des recherches sur la personne humaine comme fragile et interdépendante, et sur la relation entre philosophie et foi. Email: marie.walckenaer@gmail.com
} 
how Gabriel Marcel articulates our experience of incarnation with our reckoning of a transcendence, as opposed to a reductive materialism.

Keywords: Freedom. Grace. Creative testimony. Person. Being.

\section{Introduction}

Comme le bon philosophe décrit par Platon dans le Phèdre ${ }^{2}$, qui travaille en cherchant les articulations tel un boucher, Gabriel Marcel explore articulations et jointures: dans de telles articulations se situe la complexité de l'existence humaine. On trouve ainsi chez lui des réflexions sur les articulations de l'être et de l'existence, de la liberté et de la nécessité, du vital et du spirituel, de la situation et de l'aspiration, ou encore une analyse du sentir "à la jointure de la sensation et du sentiment" - et ainsi de suite ${ }^{3}$. Nous nous attacherons ici à "l'articulation entre la liberté et la grâce", que l'on retrouve à plusieurs reprises chez notre auteur dans les analyses de l'expérience de la liberté. Le problème classique de la liberté dans une théologie de la grâce est de maintenir ensemble l'affirmation de la liberté et celle de la grâce du salut: comment dire l'homme libre si c'est par grâce qu'il est sauvé? En effet, si la liberté ne concerne pas la destinée éternelle de la personne, la liberté est bien insignifiante et la réflexion sur celle-ci bien inutile, puisque le sens même de la vie échappe à l'action humaine. C'est pourquoi une théologie de la grâce a à se défaire sans cesse d'une double tentation: celle du pélagianisme et celle du jansénisme, qui tous deux mettent en concurrence grâce et liberté. Qu'est-ce que la grâce? Chez Marcel, elle peut être définie comme un "irréductible" qui se donne, que je ne peux pas me donner à moi-même. Eclairé par la Révélation chrétienne, mais cherchant à penser sur son seuil, à partir de l'expérience, Gabriel Marcel parle le plus souvent de don, sans faire intervenir l'idée d'une grâce au sens théologique. Sa perspective philosophique permet de poser à frais nouveaux le problème, en déplaçant le point de départ de la réflexion: il part de l'expérience de l'acte libre, et non de l'affirmation de l'existence de la grâce. Pourquoi la réflexion sur ce qu'on reconnaît comme des actes libres aboutit-elle à l'idée que notre liberté s'articule à un don préalable qui la suscite, lui donne sens et la soutient? Peut-on penser une capacité de l'homme à agir librement en faisant l'économie d'un recours à une transcendance?

Notre but sera de montrer comment la notion marcellienne d'attestation créatrice permet de lier la reconnaissance de la grâce à la reconnaissance de la liberté d'un acte, tout en donnant un contenu à la liberté. Cependant, comment tenir ensemble l'attestation et la création dans l'acte? Témoigner, n'est-ce pas par

\footnotetext{
${ }^{2} C f$. Phèdre (265e).

${ }^{3}$ Nous pouvons noter déjà que le terme "d'articulation" permet d'échapper au langage de la causalité. Cf. par exemple Le Mystère de l'Etre, vol. II, p.134.
} 
définition un acte conservateur? Créer, n'est-ce pas par définition faire surgir ce qui n'était pas déjà là, et donc s'écarter d'une simple attestation? La tension entre attestation et création éclaire la compréhension de l'action humaine, comme Gabriel Marcel l'affirme lui-même dans l'introduction de l'Essai d'une philosophie concrète:

\begin{abstract}
Au départ de toute création visible ou non, on découvre la même présence, et, ajouterai-je, la même sommation de l'être à l'âme qu'il investit, mais aussi l'acte, identique en ses spécifications infinies, par lequel l'âme rend témoignage à cette même présence qu'il lui est au reste donné de pouvoir récuser, c'est-à-dire annuler, dans la mesure même où elle est âme, c'est-à-dire liberté (MACEL, 1999, p. 20).
\end{abstract}

Les lignes qui suivent sont un commentaire de ce passage, dans lequel Gabriel Marcel lie attestation, création, présence gracieuse et liberté.

\title{
Attestatio créactrice
}

Tâchons d'abord de comprendre ce qu'entend exactement Gabriel Marcel par "attestation créatrice", puisque celle-ci est pour notre auteur le paradigme de tout acte libre. Parler d'attestation créatrice est supposer l'attestation d'un donné préalable à l'attestation, même quand l'attestation découvre celui-ci en se faisant, et une création, autrement dit "le passage d'un moindre être à un plus être" (MACEL, 1997, vol. I, p. 146). Par ailleurs, puisque la question de la liberté est en jeu "dans la mesure où un être est appelé à décider de soi” (MACEL, 2007, p. 39), il semble qu'il y ait dans l'attestation créatrice une décision qui porte sur soi et qui entraîne le passage à un "plus être".

La première condition d'une attestation est que quelque chose soit reçu que l'on puisse attester. Attester, en effet, c'est témoigner de la réalité ou de la vérité de quelque chose. Gabriel Marcel entend par une telle réception une activité, loin de l'impression passive de la cire par le sceau. Une telle activité est un accueil: l'accueil préalable à l'attestation ressemble à l'accueil de l'hôte. Accueillir véritablement suppose de s'ouvrir à celui qui vient, et mieux, voir en lui un don qui m'est fait, comme dans la tradition orientale de l'hospitalité (MACEL, 1999, p. 47). Plus encore, accueillir est alors recevoir l'autre comme don et réciproquement lui donner son intimité. C'est pourquoi recevoir est un acte de participation. Par conséquent, seul peut recevoir celui qui est doué d'une "responsivité intérieure", puisque ce qui se donne se présente comme appel, ne serait-ce que l'appel à être reçu. La présence de ce que je reçois m'atteint "du dedans" et non "du dehors" (MACEL, 1999, p. 40), et l'on comprend alors que pour Gabriel Marcel, être c'est être avec, d'une manière qui transcende la séparation du sujet et de l'objet, du pour soi et de l'en soi. Une telle séparation est le fruit de l'esprit d'abstraction, qui par une démarche d'objectivation, n'est capable que de saisir des relations de type savant ou technique. Je ne peux pas 
attester ce qui ne me concerne pas réellement, et en cela, je ne peux pas attester ce qui coexiste simplement avec $\mathrm{moi}^{4}$. La raison d'une telle abstraction est l'oubli de la condition de possibilité de toute expérience, à savoir ma condition d'être incarné. Mon incarnation, c'est-à-dire le fait que j'aie un corps sans pouvoir me réduire à lui ${ }^{5}$, est par là même le fait "d'être au monde", de telle sorte que j'y participe et que cette participation soit le mode de "ma présence au monde" (MACEL, 1999, p. 36-38). En effet, on peut renvoyer ici aux analyses par lesquelles Gabriel Marcel montre que le fait d'avoir un corps implique que j'entre en relation avec le monde par la sensation, et que même sentir est "en quelque façon recevoir" (MACEL, 1999, p. 47), et que ce recevoir est actif. Ainsi, la condition de l'attestation est qu'il y ait quelque chose à attester, et pour cela, que je le reçoive, que j'y participe, que je sois avec lui dans une forme de communion. On en trouve une illustration avec l'exemple de la Vue de Delft de Ver Meer donné par Gabriel Marcel dans Le Mystère de l'Etre. Selon Marcel, Ver Meer a pu peindre cette Vue en tant qu'il a su participer au paysage, c'est-à-dire qu'il l'a contemplé, et qu'il ne l'a pas regardé dans une position de simple spectateur depuis la rive opposée (MACEL, 1997, vol. I, p. 150). Contempler, "c'est se recueillir en présence de [...]" (MACEL, 1997, vol. I, p. 142), c'est la réalisation d'un coesse entre celui qui contemple et ce qu'il contemple. Le regard contemplatif permet à la fois de se tourner intégralement vers quelque chose tout en faisant qu'on se retourne vers soi. Il réalise le dépassement de l'opposition entre dehors et dedans. Alors que la position de spectateur implique un face à face, une frontalité, elle est l'opposé d'une participation et, on le verra, s'oppose donc à toute création. Mais plus encore, on comprend que l'attestation suppose déjà un choix, puisque recevoir est actif: le choix entre recevoir ou refuser de recevoir ce à quoi je peux être présent.

Puisque l'attestation suppose une présence à ce que j'atteste qui est participation et communion, elle est un acte dans lequel mon être lui-même est engagé, mis en jeu, et pas seulement ce que j'atteste. En cela, "dans l'attestation, je me lie moi-même, mais en toute liberté” (MACEL, 1935, p. 315). L'attestation porte sur soi tout autant que sur ce que j'atteste, par la reconnaissance du lien qui est "ma présence au monde". Toute attestation est alors un engagement; elle suppose une double fidélité, à soi et à ce qui a été reçu. Inversement, le refus de témoigner ou le fait de revenir sur un témoignage est une trahison de ce dont on témoigne, mais trahison de soi aussi, puisque l'on renie ce qui nous constitue comme étant présent au monde. Cette dimension distingue l'attitude de l'homo spectans et de l'homo particeps (MACEL, 1997, vol. I, p.138), celui-là se caractérisant par son esprit d'abstraction et de séparation, et celui-ci par sa disponibilité, c'est-à-dire "son

\footnotetext{
${ }^{4}$ Autrement dit, ce qui est pour moi simplement objet: "ce qui ne tient pas compte de moi, ce pour quoi je ne compte pás" (MACEL, 1999, P. 53) et inversement. Ou encore: "ce qui est placé devant moi en face de moi” (MACEL, 1997, vol. I, p.55).

${ }^{5}$ Etre incarné, "c'est s'apparaître comme corps, comme ce corps-ci, sans pouvoir s'identifier à lui, sans pouvoir non plus s'en distinguer" (MACEL, 1999, p. 34).
} 
aptitude à se donner à ce qui se présente et à se lier par ce don" (MACEL, 1998, p. 28). L'homme n'est pas seulement sapiens ou faber, il est surtout celui qui peut s'ouvrir à ce à quoi il participe ${ }^{6}$. Par là même, le témoignage est "une participation et une confirmation" (id.). Confirmation au sens propre, c'est-à-dire une contribution "à la croissance ou à l'avènement de ce dont on témoigne" (MACEL, 1998, p. 283). On met à la disposition de celui-ci ce qu'on est, puisqu'en l'incarnant. C'est pourquoi c'est un acte libre, un engagement, car en attestant une réalité qui m'est donnée, je m'y lie, je m'engage à reconnaître que ce ne peut pas être indifférent pour moi d'avoir été présent à cette réalité. Je ne peux pas me comprendre indépendamment de ce à quoi j'ai été présent: le sujet ne se comprend pas à partir de sa séparation d'avec l'objet. Ou plus exactement, un tel sujet est justement celui qui n'accomplit pas sa dimension de sujet ${ }^{7}$.

Or l'attestation est nécessairement créatrice selon Gabriel Marcel. Pourquoi? La création n'est pas la production, même si la création artistique est l'exemple favori de Marcel, puisque, comme il l'explique lui-même, cet exemple lui permet de faire germer et d'éprouver sa pensée (MACEL, 1997, vol. I, p. 133). Notons que même la production d'une œuvre n'est pas pour autant une création ex nihilo, puisqu'elle est le fruit de la disponibilité de l'artiste envers ce qui semble s'imposer à lui tout en dépendant totalement de lui pour s'incarner ${ }^{8}$. L'accent n'est pas mis sur le fait qu'il y ait un objet créé par l'artiste, mais sur la continuité indiscernable entre la participation dans l'artiste au monde et la création, continuité par exemple chez Ver Meer entre sa présence à Delft et le geste de la peindre. "Recevoir activement, c'est déjà créer", et inversement "tout créateur est quelqu'un qui répond"; et la réponse est déjà simplement le fait de se reconnaître participant d'une réalité qui nous tisse. Devenir créateur est une vocation, pour l'artiste sous le mode de l'œuvre d'art, pour tout homme par l'attestation créatrice du don qui le suscite. Réponse et non causalité; comme dans les notes d'une mélodie, la note précédente appelle la suivante, sans pour autant la causer. Une note qui succède à une autre n'est ni l'effet de la précédente, ni l'effet d'une conjugaison de causes dont la précédente. Il y a création justement par ce qui reste inexplicable et apparemment contingent, et qui pourtant n'est pas arbitraire, mais suscité. L'œuvre d'art se présente avec un caractère de nécessité par là: une nécessité en soi, et non un déterminisme, car dire que l'œuvre est déterminée reviendrait finalement à rendre celle-ci liée à la

\footnotetext{
${ }^{6}$ Cf. MACEL, 1935, p. 140: "L'essence de l'homme ne serait-elle pas d'être un être qui peut témoigner?"

${ }^{7}$ Cf. MACEL, 1959, p. 162: "le pour soi ne peut être entendu que comme participation: exister, c'est coexister". Il y a une critique constante chez Gabriel Marcel de la pensée du sujet comme un pour-soi séparé qui constituerait la "facticité de l'en-soi", comme Sartre le fait dans L'Etre et le Néant. Au contraire, parce que le lien entre les deux n'est pas contingent, on sort de la contingence pour l'existence de l'un comme pour l'existence de l'autre.

${ }^{8} c f$. les récits d'expérience de création dramatique ou musicale que donne Marcel.

${ }^{9}$ Ou encore, à propos de ce que Rilke" nous enseigne": "il existe une réceptivité qui est un autre nom de la création elle-même. L'être le plus authentiquement réceptif est l'être le plus essentiellement créateur" (MACEL, 1998, p. 337).
} 
contingence de l'existence de la cause ${ }^{10}$. De la même manière, on ne peut pas dire que c'est parce que Ver Meer a grandi et vécu à Delft qu'il a peint la Vue de Delft de même que ce n'est pas parce qu'il a étudié, assimilé la peinture de ses prédécesseurs Rembrandt, Van Loo, Le Caravage ni parce qu'il a été influencé par eux qu'il a été à la hauteur de ses maîtres.

L'attestation est toujours créatrice, même lorsqu'il s'agit d'un simple témoignage, sans production. Il y a création, parce qu'il y a passage d'un "moindre être à un plus être" dans le fait même de s'engager par l'attestation, de se lier - et c'est par là qu'il y a liberté. Ce qui est d'abord transformé par l'attestation, c'est l'être de celui qui s'engage dans l'attestation; la création dont il est question ici est d'abord "une création de soi par soi" (MACEL, 1997, vol. II p. 118), puisque l'attestation signifie une déprise, une ouverture à, et par là un accès à ce que c'est qu'être $^{11}$. Dès lors, on comprend que pour Gabriel Marcel un acte d'attestation, parce qu'il est auto-créateur, est le modèle d'un acte libre. Mais inversement, tout acte libre est une réponse, une attestation.

[...] on ne peut parler de liberté que là où l'enjeu [du choix] présente (...) une importance réelle. Mais cette valeur de l'enjeu n'existe pas en dehors de la conscience, ce qui ne veut d'ailleurs nullement dire, comme on serait quelquefois porté à le déclarer inconsidérément, qu'elle soit créée par la conscience. La vérité paraît être bien plutôt celle-ci: j’ai à reconnaître in concreto que je me trahirais ou que je me renierais moi-même si je ne posais pas cette valeur. Dans cette perspective il faut dire que l'acte libre est essentiellement un acte significatif. [...]. Sans doute pourrait-on dire que le propre de l'acte libre consiste en ce qu'il contribue à me faire ce que je suis et comme à me sculpter, au lieu que l'acte contingent ou insignifiant, l'acte qui pourrait aussi bien être accompli par n'importe qui, ne contribue en rien à cette sorte de création de moi-même par moimême (MACEL, 1997, vol. II p. 117-118).

L'attestation créatrice est le modèle de l'acte libre, puisque tout acte libre est finalement l'accueil - donc l'attestation -, ou le refus - donc la trahison -, de quelque chose qui m'est donné, et que je ne peux pas me donner à moi-même. D’après le texte cité ci-dessus, on voit que ce qui est donné prend la forme d'une valeur, au sens de quelque chose qui doit valoir pour moi de telle sorte qu'y renoncer serait me trahir moi-même. Du fait de la situation dans laquelle je suis ${ }^{12}$ et du fait de ce que je suis, il n'est pas indifférent que j'agisse d'une manière ou d'une autre. La valeur n'est pas un absolu indépendant de ce que je suis, elle est plutôt liée à ce que veut dire être: défendre la justice dans telle situation sera accomplir tel acte qui me libérera des tentations d'une posture d'avoir. C'est pourquoi la valeur n'est

\footnotetext{
${ }^{10}$ Cette articulation de la nécessité et de la liberté est vraie de toute attestation.

${ }^{11}$ Avoir, prendre, posséder étant la défiguration de l'être, son mouvement opposé.

${ }^{12}$ Ma situation est "ce en quoi je suis impliqué” (MACEL, 1997, vol. I, p. 15).
} 
pas dissociable de la situation dans laquelle je suis appelé à l'incarner, ce serait en faire un absolu et oublier qu'elle n'est que la direction dans laquelle je suis appelé à agir dans une situation donnée - dans la mesure même où je suis appelé à être -: "le propre de l'acte de transcendance est d'être orienté" (MACEL, 1999, p. 207). Certes, ces valeurs ne sont pas aléatoires, car être a un sens non équivoque. En cela, je ne crée pas la valeur selon laquelle mon action va s'ordonner, car je ne me donne pas à moi-même ce que je suis, ou plus exactement, ce que veut dire être: il y a là quelque chose qui me précède, qui précède mon action, une transcendance de la valeur par rapport à mon choix, et indissociablement, un appel à être qui ne dépend pas de ce que je veux être, autrement dit d'un projet. Donc ce qu'il s'agit d'attester est une valeur, mais attester cet appel en y répondant est incarner mon propre être. Ainsi, Socrate qui choisit de rester fidèle à la vérité devant ses juges, choisit de répondre à ce qui s'impose à lui comme étant la vérité. Il ne choisit pas lui-même que la justice soit dans la loyauté, dans la situation dans laquelle il est, il peut seulement reconnaître une alternative en termes de fidélité ou de trahison, et donc incarner ou non ce qu'être juste est dans cette situation - et, ô vertu de souplesse!, ce qu'être juste est ne se laisse pas séparer de la situation qui l'enjoint personnellement et donc ne se laisse pas enfermer par un discours abstrait dans une idéologie ${ }^{13}$. En ce sens, il est vrai de dire qu'aucune valeur n'est absolue, une valeur n'a de sens qu'en tant que "réfraction irisée de l'être", selon une expression des Hommes contre l'Humain. Être fidèle à soi-même, c'est accueillir la transcendance des valeurs, qui sont autant d'appels de l'être. En répondant à "l'appel" entendu dans la situation présente, je me hisse à ce que je suis dans mon être en l'incarnant dans ma vie, puisque c'est moi qui entends cet "appel" ${ }^{14}$. C'est dire corrélativement que ce que je suis ne coïncide pas avec "ma vie" présente et passée. "L’homme passe l'homme", pour reprendre le mot de Pascal, parce qu'il s'adosse dans son être à autre chose qu'à l'ensemble des actes qu'il a accomplis ${ }^{15}$, ce qu'il est est en espérance, à venir. Dans Les Hommes contre l'Humain, Gabriel Marcel revient plusieurs fois sur l'articulation de la liberté à la transcendance des valeurs. Si je pose moi-même les valeurs, autrement dit si les valeurs sont relatives, alors la liberté est doublement piégée. Tout d'abord parce que rien ne m'assure que le choix que je fais des valeurs n'est pas contraint - par le désir ou la crainte, ou par des idéologies et des opinions. Ensuite, parce qu'au niveau social, plus rien ne fait barrière à ce que les divergences d'opinions deviennent des rapports de force ${ }^{16}$. Aussi la liberté est-elle "d'abord et avant tout le pouvoir de s'affirmer ou de se nier elle-même” (MACEL, 1999, p. 44).

\footnotetext{
${ }^{13}$ Gabriel Marcel justifie ainsi ses prises de position nuancées lors des épurations après la guerre, dans En chemin vers quel éveil.

${ }^{14} C f$. l'ensemble de l'article "Mon corps, ma vie, mon être", in Présence de Gabriel Marcel, 2007, ${ }^{\circ} 17$.

${ }^{15}$ On voit combien l'opposition entre Gabriel Marcel et Sartre est constante, dans la mesure même où le point de départ est inversé chez Sartre.

${ }^{16}$ Cf. MACEL, 1951, p. 32: "Mais ce qu'il faut proscrire sans pitié, c'est l'illusion d'après laquelle le mot liberté peut conserver une signification quelconque là où le sens des valeurs a disparu; et il faut
} 
Tout acte libre est une attestation, car il est réponse à un appel à être qu'il révèle par là-même. Une telle réponse est libre car elle est créatrice. Or "c'est en tant que créateur, si humble que soit le niveau où cette création se consomme, qu'un homme quel qu'il soit peut se reconnaître libre" (MACEL, 1951, p. 24). Dire qu'il y a création, c'est dire qu'il y a par l'acte, ou plutôt dans l'acte, un plus-être, une plusvalue ontologique, si l'on peut dire. Je suis libre pour autant que ma vie a un enjeu: m'ouvrir à l'être, me situer dans la "dimension de l'être"17 ou m'en séparer ${ }^{18}$. Dans notre existence se joue "l'intime articulation de l'être et de la liberté" (MACEL, 1999, p. 88). On retrouve ici l'idée que mon être est l'enjeu de ma vie ${ }^{19}$. C'est-à-dire que de la liberté d'un acte dépend l'enjeu en termes de développement de la personne: je suis une personne dans l'exacte mesure où je ne suis pas hors de moi-même, aliéné à l'avoir. C'est pourquoi la liberté fait de l'existence une épreuve, au sens propre: la liberté intervient “à la jointure de l'être et de l'existence” (MACEL, 1997, vol. II p. 31). Regardons ce qui se passe dans le refus de répondre à ce à quoi la situation m'invite. Quel est le ressort d'une telle "trahison"? La crainte ou le désir, qui me font traiter ma vie comme un avoir, que je possède et donc que je pourrais perdre ou gagner selon des images que la crainte et le désir forment en moi, au lieu de m'ouvrir à ce qui ne vient pas de moi $^{20}$. Il s'agit alors d'une "pulvérisation du sujet" dans l'avoir, serait-ce celui de sa propre vie. Par le désir et la crainte, le centre de gravité de la personne se déplace de ce qui est possédé, et la personne s'émiette, pour ainsi dire ${ }^{21}$. Qu'il y ait perte possible fait comprendre corrélativement qu'il y a un gain possible, qu'il y ait chemin, quand l'acte me libère de cet émiettement, même si je ne le sais pas d'avance: “dire qu'il est libre, c'est dire qu'il ne sait lui-même qu'après l'acte, ou après l'épreuve, ce qu'il est, c'est-à-dire ce qu'il portait en lui” (MACEL, 1964, p. 9). "Décider de soi" est donc possible d'abord dans l'alternative d'une intégrité ou d'une pulvérisation de soi, d'un développement ou non de sa dimension personnelle. En effet, le propre de la personne, écrit Marcel, consiste "à affronter directement une situation donnée et [...] à s'engager effectivement" et donc "à collaborer avec son propre destin en lui conférant sa marque propre” (MACEL, 1998, p. 25) ${ }^{22}$. La création

entendre par là le sentiment de leur transcendance. On pourrait dire sans paradoxe quà l'heure présente c'est d'une cure de platonisme que les hommes ont le plus grand besoin". On ne saurait être plus clair sur les maux de notre époque: une perte du sens de la liberté!

${ }^{17} C f$. "Mon corps, ma vie, mon être", in Présence de Gabriel Marcel, 2007, nº 17, p. 18.

${ }^{18}$ Gabriel Marcel nomme "mon âme" le fait qu'il y ait un enjeu, ce qui en est une définition originale mais suggestive. $C f$. MACEL, 1935, p. 127.

${ }^{19}$ Cf. MACEL, 1935, p. 291. Ou encore MACEL, 2007, p. 34: ma vie est "le fait d'être au monde avec toutes ses implications concrètes".

${ }^{20}$ Gabriel Marcel désigne sous le terme de désir le désir sous sa forme aliénante, par opposition justement à ce qui serait l'aspiration née d'une vocation.

${ }^{21}$ On pourrait penser ici à ce qui se passe dans la sortie de la caverne dans l'allégorie de Platon: le passage d'un émiettement dans l'image à une unification dans la vision - si ce n'est que chez Marcel, l'intégrité est en espérance, elle n'est pas en arrière de soi.

${ }^{22}$ Ajoutons que la caractéristique essentielle de la personne est la disponibilité, alors que celle du sujet "pulvérisé" dans l'avoir est l'indisponibilité, puisque la disponibilité est une "aptitude à se donner à ce qui se présente, et à se lier par ce don” (MACEL, 1998, p. 25). 
est véritable, même si elle est une réponse, car seul moi pouvais donner la réponse que je donne: elle est personnelle $e^{23}$. La personne désigne ce que je suis dans l'acte où je m'engage, où je m'expose. Gabriel Marcel identifie ici être une personne, progresser dans la direction de l'être, accomplir sa dimension de sujet.

C'est pourquoi si l'acte est libre quand je décide de mon être, je ne peux séparer l'être et la liberté que par une démarche de la raison objectivante: la liberté n'est pas une de mes facultés ni une de mes qualités. Mon acte n'est pas libre quand il a été choisi librement, mon acte est d'autant plus libre qu'il me fait agir par l'intégralité de mon être; par là j'incarne dans ma vie mon être, je suis. L'attention à la liberté est école d'unification - car elle est attention à ce que je suis ainsi qu'au réel: la liberté est "le mystère central de mon être". Retrouver le lien de ma "présence au monde", paradoxalement, me garde de l'émiettement et de la pulvérisation ${ }^{24}$, alors même qu'on aurait pu penser que ce serait me faire sortir de moi-même pour me perdre en m'aliénant à une extériorité. Ainsi, la liberté n'est pas désir - et Gabriel Marcel reprend les termes du débat entre Socrate et son adversaire Calliclès, qui confond liberté avec puissance ${ }^{25}$-, et elle n'est pas non plus indétermination, liberté d'indifférence, capacité à poser un choix par délibération raisonnable. Ces deux manières de comprendre la liberté n'arrivent pas à se dégager de la catégorie de la liberté comme cause de l'acte, et de ce fait même s'exposent à des critiques définitives ${ }^{26}$. Elle n'est pas non plus pouvoir d'auto-fondation, puisque l'on voit que dans l'acte libre, si je décide de moi, c'est à partir d'une vocation, au sens propre, lancée dans la situation qui est la mienne. Elle n'est pas même le moyen par lequel je deviens moi-même: être libre, "c'est être soi" ${ }^{27}$, puisqu" un de mes actes se révélera à moi-même comme d'autant plus objectivement libre que ma réalité de sujet apparaîtra elle-même comme y étant plus effectivement engagée" (MACEL, 2007, p. 27). Ma liberté peut s'éprouver exactement dans les limites dans lesquelles je peux m'éprouver comme sujet. Dans tout acte où je me trouve sujet, je sais que je suis libre.

Notons que cette création du sujet est corrélative d'un développement de la dimension d'inter subjectivité, qui est développée dès que l'on se tourne vers l'être ${ }^{28}$.

\footnotetext{
${ }^{23}$ Mais la liberté n'est pas pour autant comme chez Bergson cette "création d'imprévisible nouveauté", car elle s'articule à une transcendance, à une dimension verticale absente de la vision immanentiste de la création bergsonienne. De plus, l'appel finalise l'action, alors que Bergson se situe hors de tout finalisme.

${ }^{24} C f$. infra.

${ }^{25}$ Cf. Platon, Gorgias, 491d sq.

${ }^{26}$ La plus définitive de ces critiques étant celle de Spinoza: rien ne garantit qu'on n'est pas simplement dans l'ignorance des causes qui nous déterminent. $C f$. MACEL, 2007, p. 25.

${ }^{27}$ MACEL, 1999, p. 85: la liberté n'est pas "un moyen que nous aurions à notre disposition, comme un soporifique que nous nous procurons chez un pharmacien, ou un revolver que nous achetons chez un armurier; pour cette simple raison qu'elle n'est pas quelque chose d'autre, qu'elle est nous-même, nous-même en tant que sujet".

${ }^{28}$ Cf. MACEL 1997, vol. II, p. 20: "je ne me soucie de l'être que pour autant que je prends conscience plus
} 
En effet, la structure même du sujet est inter subjective (MACEL, 1997, vol. I, p. 198), mais surtout, ce qu'il faut remarquer pour notre propos est ici que l'inter subjectivité est la réalisation de la co-présence. Quand j'atteste quelque chose, j'accepte de l'exposer à l'autre. Mais puisque c'est ma propre "présence au monde" que j'expose, c'est donc moi-même que je livre au regard ou à l'appréhension de l'autre. J'agis alors comme si une cité idéale existait - ou plus exactement pour Gabriel Marcel j’agis déjà “dans la cité idéale” (MACEL, 1997, vol. I, p. 86)-, à laquelle nous appartiendrions tous deux. Cette cité est caractérisée par le fait que nous serions dans une communion faite d'accueil mutuel, à l'exclusion des rapports d'avoir, de prise, de possession, et donc de rivalités et autres postures de crispation sur l'avoir. Il s'agit alors d'une communion dans la transcendance, puisque ouverte à ce qui n'est à la portée de prise ni de l'un ni de l'autre ${ }^{29}$. Mais plus encore, quand je témoigne par la parole ou par la vie, je mets en présence de l'autre ce qu'il peut accueillir ou refuser d'accueillir, mais en tous les cas ce dont il ne peut pas se saisir sans corrélativement le transformer en ce qu'il n'est pas, sans le distordre: j'expose à l'autre ce qui est hors de ses prises, puisque ce n'est pas prenable. J'ouvre donc la possibilité d'une expérience de communion, parce que je la crois possible, et parce que moi-même en acceptant de répondre à ce qui m’est donné, je me donne à l'autre. Je témoigne qu'il y a un don fait à un "nous-tous", à une universalité concrète.

Dans la mesure même où être libre, c'est être soi, on peut se tromper sur sa propre liberté. En effet, cela implique que je ne peux juger de ma propre liberté que si je me connais en tant que sujet. Dès lors, celui qui est pulvérisé dans l'avoir, aliéné au désir et à la crainte, est incapable de juger de sa propre liberté, puisqu'il est coupé de sa réalité de sujet, de l'intégrité de son être. En effet, traitant sa propre vie comme un avoir, il est pour lui-même un objet. Pour la même raison, ce que Gabriel Marcel appelle la réflexion première, qui abstrait et me rend étranger à moi-même en m'objectivant, est incapable d'éclairer la notion de sujet, et par là même celle de liberté. "Ma réalité de sujet" est ce que je peux approcher de moi dans un acte d'amour, de piété envers moi-même, de la même manière que je ne peux approcher l'autre comme personne et reconnaître sa liberté que dans un acte d'amour. Plus encore, je suis libre du fait de reconnaître la liberté de l'autre - et je le suis du fait de m'approcher moi-même comme sujet ${ }^{30}$. Ainsi, un acte de reconnaissance, un don créateur même, se jouent là ${ }^{31}$. Dans cet acte d'amour, je deviens intime avec moi-

ou moins distinctement de l'unité sous-jacente qui me relie à d'autres êtres dont je pressens la réalité”.

${ }^{29}$ Il est notable que l'intersubjectivité ne soit dès lors possible que dans la lumière de la vérité. Dès qu'il y a mensonge, je me coupe de l'autre. L'universalité concrète est indissociable de la vérité.

${ }^{30}$ Cf. MACEL, 1999, p. 99: l'amour est "l'acte d'une liberté qui en affirme une autre et qui n'est liberté que par cette affirmation même. Il y a là, à la racine de l'amour, la croyance en l'inexhaustible richesse et à l'imprévisible spontanéité de l'être aimé".

${ }^{31}$ Cf. par exemple: Miklos Vetö, "La métaphysique de la paternité”, in Présence de Gabriel Marcel, $\mathrm{n}^{\circ} \mathbf{2 2}$, 2014-2015, pp.41-6o, sur la reconnaissance du fils par le père. L'amour est le contraire du désir ou de la 
même. L'intimité est distance et proximitée ${ }^{32}$. Distance, puisqu'aimer demande de sortir de soi: je me libère de l'autocentrisme dans lequel le désir et la crainte m'enferment. Choisir la "négation de tout autocentrisme", c'est pour Gabriel Marcel faire"acte de transcendance" (MACEL, 1999, p. 207). En effet, sortir de soi, en orientant son action selon d'autres motifs que le désir ou la crainte est ouvrir sa volonté à ce qui l'éclaire de plus loin qu'elle-même. Il n'est pas étonnant alors que l'on trouve comme devise du sujet sursum (MACEL, 1998, p. 32) - de sub et versum, par en-dessous vers le haut ${ }^{33}$. Proximité également, parce que cet hétérocentrisme n'en est pas un, puisqu'il me rend à moi-même en me rendant présent à moi-même dans la mesure où je renonce à m'objectiver. Autrement dit, j'entre alors dans le mystère de mon être, au sens que Gabriel Marcel donne au mystère. Par exemple, quand je m'engage, je cesse de me regarder pour me tourner intégralement vers ce envers quoi je m'engage ${ }^{34}$. C'est cependant surtout dans le recueillement, au sens propre d'un recueil, possible quand je me tourne vers moi-même, que je m'approche de ce que je suis tel que je ne peux pas me saisir, c'est-à-dire de ma vocation, de l'appel de l'être au sein de mon existence - de ce que je suis seulement en espérance.

L'alternative montrée par Gabriel Marcel est celle-ci: soit je considère que je construis moi-même ma vie, et je suis libre car je choisis d'être qui je veux. Alors tout ce qui s'oppose à la contingence de mon désir est une contrainte pour ma liberté - au risque de me perdre dans le désir et la crainte. Pour Marcel, il y a là illusion de liberté, car je ne suis pas créateur, je m'éloigne de l'être plutôt que de contribuer à son accroissement. Soit j'accueille ma vie, je m'engage à répondre aux situations dans lesquelles je suis pris, et alors je suis libre car je suis libéré des multiples aliénations qui m'empêchent d'accomplir ma dimension personnelle. L'acte libre s'articule donc pour Gabriel Marcel à la reconnaissance que je ne me donne pas à moi-même ce que je suis, même si c'est mon acte qui me fait accéder à ce que je suis parce que je suis libre, qui me "fait décider qui je suis" (MACEL, 2007, p. 39), au sens où le développement de ma personne dépend de moi. Or être capable de voir ma vie comme un don, dans sa dimension particulière, dépendante de situations le plus souvent non choisies, voire difficiles ou douloureuses, n'est-ce pas déjà un don? Il semble plutôt qu'une telle reconnaissance est plus ou moins facilitée par les conditions dans lesquelles on est placé. Ainsi, la tragédie de notre monde contemporain est de créer au contraire des conditions qui empêchent une telle

crainte: il ouvre à la confiance là où le désir et la crainte crispent dans la peur.

${ }^{32}$ Cf. MACEL, 1997, vol. I, p. 33: "La présence et la distance tout ensemble: c'est bien cette sorte de contradiction effective qui permet de définir mon rapport à mon être".

33 Gabriel Marcel affirme lui-même qu'on ne peut pas renoncer là à la métaphore spatiale de la verticalité (MACEL, 1997, vol. I, p. 48).

${ }^{34}$ On trouve la même idée chez Buber par exemple: le fait de dire Tu m'unifie, je ne peux dire Tu qu'avec toutes les dimensions de mon être. Ou alors je dis Il en disant Tu. $C f$. BUBER, 2006, pp.21sq. 
reconnaissance et qui poussent même à vouloir être au fondement de son propre être: “monde cassé”, selon l'expression d'une héroïne de Marcel, Christine ${ }^{35}$.

Le "monde cassé" est le monde de la division: dissociation entre le biologique et le spirituel - il n'est qu'à voir les polémiques sur la filiation -, entre mon corps et ce que je suis; écart entre puissance technique et responsabilité véritable face à l'autre; rupture entre égalité et fraternité; atomisation des individus dans un tout social anonyme; perte de l'intimité et de l'intériorité à la faveur du divertissement; démission devant le désir et la crainte corrélativement à la maîtrise de plus en plus grande des techniques; refus de réfléchir et d'imaginer face aux conséquences des décisions politiques, et corrélativement pouvoir des mots qui se vident de leur substance et deviennent des slogans (égalité, démocratie, personne, etc.). Monde "cassé" et terrifiant, monde de l'abstraction, que notre monde tel que Gabriel Marcel le décrit dans Le Mystère de l'Etre! Or ces divisions rendent difficile l'acte de reconnaissance préalable à l'accueil de ma vie comme un don, car ce qui est divisé se montre d'abord comme absurde ${ }^{36}$.

Il ne s'agit pas pour Gabriel Marcel de prouver que la liberté s'articule à un don préalable. Il n'y aurait pas de sens à chercher à prouver ce qui est de l'ordre du mystère: il n'est possible que d'y inviter. On sait le statut que Gabriel Marcel donne aux preuves: elles ne convainquent que ceux qui y croient déjà, car elles supposent pour être entendues d'entrer dans le mystère qui les éclaire. Marcel cherche plutôt à rendre compte de l'expérience de la liberté, et à faire appel à l'expérience de ses auditeurs: je fais l'expérience de la liberté dans des actes de libération, à savoir quand je suis face à moi-même et face au réel dans une attitude d'ouverture et d'accueil. Ces actes de libération sont des expériences de création, de croissance, d'unification ainsi que de déprise et d'incarnation. Ces actes me permettent de lire ma vie en lui conférant un sens, parce que ce que j'ai eu à vivre m’a été comme adressé. Il y a là mystère dans la double dimension qu'en donne Gabriel Marcel: lumière et intersubjectivité. Lumière car accueillir ma vie comme un don éclaire l'action, en éclaire le sens. Intersubjectivité, car répondre est donner, c'est-à-dire être davantage. Puisqu'on est à l'articulation du vital et du spirituel, une telle expérience ne donne pas lieu à un savoir transmissible: "nous sommes ici encore sur le terrain de l'éprouvé" (MACEL, 1997, vol. I, p. 226).

Or l'accueil de ce don qui me rend sujet n'est possible qu'ici et maintenant, dans la présence à moi-même. La présence ne peut être qu'accueillie, elle échappe à la prise: elle est de l'ordre du mystère. Elle me confronte d'abord à la situation fondamentale d'avoir un corps, situation condition de toutes les situations, l'ici et

\footnotetext{
${ }^{35}$ Expression reprise par Marcel lui-même dans Le Mystère de l'Etre, I, 2 ème leçon: "Le monde casse".

${ }^{36} c f$. "Mon corps, ma vie, mon être", in Présence de Gabriel Marcel n' ${ }^{17}$, 2007, p.17: "Ce qu'il faut donc reconnaître, c'est que dans un monde semblable, l'affirmation d'après laquelle la vie est un don de Dieu tend à se perdre, je ne dis pas sa plausibilité, car ce mot ne saurait s'appliquer, mais sa résonance". Notons qu'ici, l'auteur du don est nommé. $C f$. infra.
} 
maintenant dans lequel je suis inséré, et qui rend possible pour moi la présence au monde et la présence à moi-même ${ }^{37}$. C'est pourquoi l'acte premier de la liberté, acte à rejouer dans toute situation particulière, est un positionnement face à ma propre incarnation. Gabriel Marcel rejoint ici la conception de l'acte libre qui est celle du combat spirituel chez les auteurs de la tradition chrétienne: il y a un combat de la liberté, et son lieu essentiel est l'incarnation. Mais la structure de l'existence me pousse à objectiver et à me séparer du monde, et je peux choisir de nier ma participation au monde. Reconnaître ou non la réalité de mon incarnation, c'est vivre ou non ma "présence au monde" ${ }^{38}$, et dans ce choix est "l'essence même de la liberté" (MACEL, 1997, vol. I, p. 185) ${ }^{39}$. En effet, d'une part c'est finalement ce choix qui est au fond de tout acte, et d'autre part, par ma liberté j'accède alors au fait que je ne me précède pas moi-même, ou alors je me pose comme seul fondement de mon existence.

C'est ainsi qu'il y a chez Gabriel Marcel une identification de l'intériorité et de la "non-contingence du donné empirique" qui pourrait sembler au premier abord surprenante: "Mais il semble bien qu'il existe un lien étroit sinon une identité entre ce que j'ai appelé plus haut intériorité et la non-contingence du donné circonstanciel. Disons encore qu'il n'est possible de parler d'intériorité que là où ce donné circonstanciel affecte une valeur positive, c'est-à-dire contribue à un développement créateur" (MACEL, 1997, vol. I, p. 150). C'est à partir du moment où je suis vis-à-vis de la situation dans une attitude d'accueil, c'est-à-dire la traitant comme un don, c'est à partir du moment où je reçois ma vie que la possibilité de l'intériorité se constitue ${ }^{40}$ - réciproquement, Ver Meer n'aurait pas pu être le créateur qu'il a été sans Delft, le donné circonstanciel n'est pas "un habit" dont on peut se "dépouiller" (MACEL, 1997, vol. I, p. 148). A partir de là, on comprend l'inquiétude de Gabriel Marcel pour la difficulté à l'intériorité de ses contemporains qu'il lie à la société technicisée, société entraînant à une surenchère du désir et de la crainte, et partant, à la pulvérisation du sujet. Il n'est pas étonnant que chez Sartre par exemple, l'idée d'une liberté auto-fondée soit liée à l'idée de la situation dans laquelle je suis comme contingente et prenant sens, dès lors, d'un geste ex nihilo de ma liberté: "mon corps, c'est la forme contingente que prend la nécessité de ma

\footnotetext{
${ }^{37}$ Alors que chez Sartre, la liberté "n'est libre qu'en constituant la facticité comme sa propre restriction" (SARTRE, 1976, p.576). Tout ce que je ne me donne pas à moi-même m'est une contrainte, et de plus c'est moi qui constitue ma situation comme contrainte contingente.

${ }^{38}$ Cf. MACEL, 1999, p. 37-39. Notamment p. 38: "Mais alors, cette participation au monde, je ne puis l'affirmer, ou la retrouver, la restaurer, qu'en résistant à la tentation de la nier, c'est-à-dire de me poser comme entité séparée".

${ }^{39}$ Ou encore: cette liberté qui s'affirme est "une liberté qui se saisit d'abord comme simple pouvoir du oui ou du non, s'incarne, ou si l'on veut, se constitue comme puissance réelle en se conférant à ellemême un contenu au sein duquel elle se reconnait" (MACEL, 1999, p. 44).

${ }^{40}$ Et aussi dans la mesure où cet accueil contribue à un "développement créateur" (MACEL, 1997, vol. I, p. 150). Le recueil est fructueux.
} 
contingence" ${ }^{41}$. Penser mon corps contingent, fruit du hasard et de la nécessité, c'est déjà penser mon être contingent. Or, c'est s'abstraire de l'expérience et se traiter soimême comme objet de connaissance: il y a là pour Marcel un refus de l'expérience. L'expérience serait plutôt celle-ci: il est possible d'accueillir mon corps comme donné, et par là même ma vie comme donnée, dans la possibilité d'une réponse à ce don, réponse qui me donne accès à mon être. Et alors je vois que mon acte a un sens. Comme l'écrit Gabriel Marcel dans Le Mystère de l'Etre (II 117), l'être incarné est "la zone où nous avons vu s'opérer la jonction de la liberté et de la grâce". En cela, c'est le fait même d'être incarné qui rend possible ma liberté telle que je l'éprouve: mon incarnation en tant qu'expérience que quelque chose m'est donné que je n'aurais pas pu me donner à moi-même articule mon existence à quelque chose de transcendant, et ainsi rend possible que je sois libre. Si je peux être de plus en plus, c'est parce que l'appel que je peux entendre dans une situation est un appel à une libération. Or cet appel me vient de plus loin que moi-même. On le voit, Gabriel Marcel n'appelle pas grâce ce qui me fait agir, mais le don qui m'invite à agir, et qui suscite ma liberté. La structure de mon expérience doit pouvoir me permettre de reconnaître que ma liberté s'articule à un don préalable. Cependant, c'est seulement de l'intérieur de la posture de la reconnaissance que je pourrai nommer grâce d'avoir fait le choix de la reconnaissance: mystère qu'est ma vie, et qui ne se reconnaît comme mystère que lorsque j'y suis entré ${ }^{42}$. Toute interprétation causale est ici en défaut, comme le note Gabriel Marcel dans la leçon conclusive du Mystère de l'Etre ${ }^{43}$.

L'existence est doublement dramatique, car il y a un double niveau d'accueil ou de refus possible. Le premier est le niveau de l'acceptation de ma situation fondamentale, à savoir ne pouvoir m'abstraire, être ici et maintenant de telle sorte que je me précède moi-même. Le deuxième est l'acceptation des situations particulières, que je peux lire comme contingence ou comme grâce selon la manière dont je les envisage: en séparant mon être de la situation ou en acceptant le lien et en me créant dans cette acceptation. Le double refus de ce don conduit à la position selon laquelle tout est contingent, et donc rien n'a de sens sinon celui que j'invente, nécessairement arbitrairement - et l'on retombe dans le nihilisme. C'est pourquoi le nihilisme intégral est le seul cohérent, même si, note Gabriel Marcel, il n'est guère praticable dans les conditions concrètes de notre vie - et d'aucuns y verront un signe. La double acceptation est la double attestation du don qui m'est fait, et la conséquence est de lire dans les situations que je vis que “cette vie m’a été donnée

\footnotetext{
${ }^{41}$ in SARTRE, 1976, p.371.

${ }^{42} \mathrm{cf}$. le très beau texte de la conclusion du Mystère de l'Etre: "Chacun de nous est situé de façon à pouvoir reconnaître que son essence est don, et non pas donnée, qu'il est lui-même un don, et qu'en dernière analyse il n'est aucunement par soi; mais d'autre part, c'est à partir de ce don fondamental que peut se déployer cette liberté qui ne fait qu'un avec l'épreuve au cours de laquelle chacun aura à décider de lui-même" (MACEL, 1997, vol. II, p. 174).

${ }^{43}$ MACEL, 1997, vol. II, p. 178.
} 
pour que je lui confère un sens" (MACEL, 2007, p. 35). Le "pouvoir réverbérateur des faits" (MACEL, 1997, vol. II, p. 150) laisse ce sens ouvert, et le présent ouvre de nouveaux horizons de sens à mes situations et actes passés. L'unité se construit peu à peu, et l'attestation au présent crée ce que je suis au présent, mais influe encore sur le sens du passé. Il n'est qu'à voir par comparaison la liberté de ceux qui n'ont pas de corps, la liberté des anges! Liberté d'un unique choix, car il n'y a pas de réfraction dans de multiples situations singulières par l'incarnation et la temporalité qui lui est liée. L'enjeu de la liberté est pour eux patent, il leur manque l'obscurité de l'expérience d'incarnation, eux dont on dit qu'ils sont à la face même de Dieu. Ils sont face au don qui leur donne l'être, c'est-à-dire qui les fait don, et accueillir une fois ce don est l'accueillir sans cesse. Les anges n'ayant pas de corps, ils ne peuvent pas vivre ce que je vis de l'écart entre "ma vie" et ce que je suis, écart dans lequel ma liberté joue sans cesse et toujours à nouveau le sens de mon existence, en me rendant peu à peu collaborateur de la création de mon être. Penser la liberté, pour l'homme est penser l'incarnation, non comme obstacle, mais comme ouverture à une transcendance.

Il reste évidemment à poser une question: s'il y a don, qui donne? La réflexion permet de passer du donné à une donation, peut-elle nous mener au donateur? Autrement dit, ma vie et mon être, et la possibilité de les reconnaître comme dons, cela est-il Grâce, au sens traditionnel de la théologie? Ce qu'il est déjà possible de répondre, c'est qu'on ne peut pas, si l'on est rigoureux, nier qu'il y ait un donateur. En effet, ce serait supposer que je suis ici dans un domaine où je peux identifier une cause, et vérifier son statut de cause par rapport à l'effet que je considère. Autrement dit, ce serait traiter cette question comme un problème susceptible d'être résolu. Or on est ici dans le non-vérifiable, car on est dans le "nonidentifiable”. Parler de non-identifiable n'est pas ici ruse du croyant pour éviter d'avoir à se confronter en toute honnêteté aux objections de l'athée, qui affirme "qu'il n'y a personne", ni illusion du "moi orant" démasquée par le "moi réfléchissant" (MACEL, 2007, p. 34). Au contraire, Gabriel Marcel montre que c'est l'analyse rigoureuse de l'expérience du don qui y mène. Reprenons les grandes étapes de son raisonnement dans l'article "Don et liberté": s'il y a don, on peut reconnaître qu'il y a générosité. Mais la relation entre la générosité et le don n’est pas causale. Il faut plutôt dire que la générosité est "l'âme du don", son "essence active". Le lien entre la générosité et le don n'est ni un phénomène mécanique, comme l'écoulement d'un trop plein, ni un phénomène finalisé. La comparaison qui semble la plus juste est celle de la lumière: la générosité est comme une lumière qui serait éclairante pour soi comme pour autrui, dans un dépassement de la limite entre le pour-soi et le pour-autrui; elle rayonne. Et Gabriel Marcel de donner cette belle définition: "une lumière qui serait joie d'être lumière" (MACEL, 2007, p. 31). De plus, la générosité ne peut pas être le résultat d'une action pour être généreux, ni d'un calcul. Elle échappe elle-même à une relation de causalité, elle est elle-même 
don, "éclat ou jaillissement" (MACEL, 2007, p. 33). Alors, si je peux accueillir ma vie comme un don, je peux croire que "mon être-au-monde se révèle à moi comme l'expression d'une générosité qui prend en quelque sorte corps en lui”, dans l'ouverture à un "Toi absolu” (MACEL, 2007, p. 36) qui se laisse reconnaître luimême comme un don qui serait à lui-même son propre principe. Et de même pour la liberté. Sans que l'on puisse parler pour autant de causalité, on peut dire comme on l'a vu précédemment qu'il y a liberté quand il y a création, qu'il y a création quand il y a réception active d'un don, qui est une participation. Alors, de même que la générosité est "l'essence active" du don, de même je peux affirmer que la Grâce est "l'essence active" de la liberté - à condition bien sûr de reconnaître que je suis libre $^{44}$. En deçà de la Révélation, je ne peux nommer Dieu. Je peux néanmoins témoigner que "la situation fonctionne comme un appel" (MACEL, 2007, p. 40), appel de ce qui en moi peut rayonner à ce qui ne rayonne pas encore, appel à ce qui est en moi analogue à une générosité. En suivant ce chemin dans lequel la réflexion s'affranchit des schèmes de la causalité, je peux approcher le mystère de l'articulation et de la collaboration de ma liberté et d'une grâce.

La philosophie de Gabriel Marcel permet alors de penser le mystère ontologique à partir de l'expérience de la liberté. Puisque les hommes libres sont des hommes qui témoignent de l'appel à être, on peut approcher concrètement ce qu'être veut dire dans l'élucidation des expériences d'accueil que sont l'amour, la fidélité et la foi, l'espérance ${ }^{45}$. La fidélité, par exemple, à l'inverse de la trahison de soi et du mensonge, entraîne une plus-value ontologique qui est une approche: "vivre dans la fidélité, c'est progresser dans la direction qui est celle même de l'Etre" (MACEL, 1964, p. 93). Dans l'élucidation de la foi, qui est la réponse ultime à la vie comme don et comme appel à être, Gabriel Marcel signifie clairement le lien entre liberté et être. En effet, l'engagement envers Dieu est un "acte de transcendance avec contre-partie ontologique qui est la prise de Dieu sur moi. Et c'est par rapport à cette prise que ma liberté s'ordonne et se définit" (MACEL, 1935, p. 76). En effet, la foi est une confiance inconditionnelle envers le "Toi absolu”, confiance qui engage tout l'être, dans son intégralité, en le rassemblant dans l'acte de foi ${ }^{46}$. La foi est donc accueil inconditionné. La vie m’est donnée, et si je la reçois, je m’en déprends. Or être, c'est donner, puisque perdre dans l'ordre de l'être, c'est prendre et posséder. C'est pourquoi l'ordre ontologique est connu "personnellement par la totalité d'un être engagé dans le sien drame de s'ouvrir à l'Etre ou de le nier" (MACEL, 1935, p. 175), c'est-à-dire de s'ouvrir à ce qui donne et est au principe de tout don - de

\footnotetext{
${ }^{44}$ Personne ne peut le reconnaître pour moi, on n'est pas dans l'ordre d'un savoir possédé, ce qui supposerait un savoir indépendant de celui qui sait. Cette reconnaissance de ma propre liberté est une croyance, une action où je décide de ma propre liberté. $C f$. MACEL, 2007, p. 39: il y a un poids ontologique de l'expérience humaine, qui correspond à "la charge d'amour dont l'expérience humaine est susceptible".

${ }^{45}$ Cf. MACEL, 1935, p. 149; MACEL, 1964, p. 110.

${ }^{46} C f$. MACEL, 1999, p. 197.
} 
toute réponse. L'ontologie ne peut faire l'objet d'un discours impersonnel et savant, le philosophe peut seulement contribuer à ce que résonne l'appel à entrer dans le mystère de l'être, qui est un appel à croire, c'est-à-dire finalement à s'incarner.

\section{Conclusion}

Nous pouvons de ce parcours conclure que la notion d'attestation créatrice permet de donner un contenu concret à la liberté, alors même qu'on renonce à la catégorie de la causalité, catégorie sous laquelle on la pense ordinairement. Elle déplace la compréhension de la liberté du problème de l'insertion d'une causalité libre dans la série des phénomènes, au mystère d'une profondeur de l'expérience où l'acte se reconnaît comme acte de participation. Et ce, parce qu'elle permet de lier la liberté à notre situation fondamentale d'être incarné, par laquelle notre corps n'est ni l'obstacle de la liberté comme chez Platon par exemple, ni le lieu contingent et accidentel de notre action dans le monde tel que Sartre le comprend. L'incarnation par laquelle je suis mon corps sans m'y réduire est l'expérience d'un écart. Si l'on n'y voit pas un don et un appel grâce à une certaine qualité de présence à soi, au monde, aux autres, on se met peut-être dans la nécessité d'appréhender tout ce qui me viendrait de l'extérieur de mon désir comme menaçant ma liberté, si ce n'est contraire à elle. Pour qu'une articulation bouge, il faut du jeu, et ce jeu est permis par la confiance par laquelle nous entrons dans le mystère. Le refus de l'ordination de la liberté à un don qui la précède finit par disjoindre l'articulation, et donc la possibilité d'un mouvement libre. A l'autre extrême, le mystère est accueilli sans réserve par une confiance donnée: la sainteté permet un mouvement parfaitement souple. Pour le saint, tel le curé du Journal d'un curé de campagne de Bernanos que cite Marcel dans le Mystère de l'Etre ${ }^{47}$, "tout est grâce", et donc tout est liberté.

\section{Références}

BUBER, Martin. Je et Tu. Trad. G. Bianquis. Aubier, Bibl. Philosophique, 2006.

MARCEL, Gabriel. La Dignité humaine et ses assises existentielles. Paris: Aubier, 1964. . Etre et avoir [1928-1933]. Paris: Aubier, 1935.

. Essai de philosophie concrète, coll. Folio Essais: Gallimard, 1999.

. Les Hommes contre l'Humain, Paris, Ed. du Vieux Colombier, 1951.

. Homo Viator. Prolégomènes à une métaphysique de l'espérance. Editions Présence de Gabriel Marcel, 1998.

. Le Mystère de l'Etre, vol. I et vol. II. Editions Présence de Gabriel Marcel, 1997.

Présence et Immortalité. Journal métaphysique 1938-1943 et autres textes.

Flammarion, coll. 10-18, 1959.

.Don et liberté", in Présence de Gabriel Marcel, n¹7, 2007, pp.25-43.

\footnotetext{
${ }^{47}$ Bernanos cite lui-même sainte Thérèse de Lisieux.
} 
BAHUREL, M.

SARTRE, Jean-Paule. L'Etre et le Néant. Gallimard, coll. Tell, 1976.

Submissão: 15. 12. 2017 / Aceite: 15. 01. 2018. 